\title{
Personal time and psychopathology
}

\section{Gabriel Roseanu*1, Mihai Marian¹, Ioana Mihaela Tomulescu² and Claudia Teodora Pusta ${ }^{3}$}

Address: ${ }^{1}$ Department of Psychology, Faculty of Social and Humans Sciences, University of Oradea, Oradea, Romania, ${ }^{2}$ Department of Biology, Faculty of Sciences, University of Oradea, Oradea, Romania and ${ }^{3}$ Department of Anatomy, Faculty of Medicine and Pharmacy, University of Oradea, Oradea, Romania

* Corresponding author

from International Society on Brain and Behaviour: 3rd International Congress on Brain and Behaviour Thessaloniki, Greece. 28 November - 2 December 2007

Published: 17 April 2008

Annals of General Psychiatry 2008, 7(Suppl I):S269 doi:I0.II86/I744-859X-7-SI-S269

This abstract is available from: http://www.annals-general-psychiatry.com/content/7/SI/S269

(c) 2008 Roseanu et al.; licensee BioMed Central Ltd.

\section{Background}

Time plays an essential role in differentiating among individuals. Each person has a characteristic way of experiencing time and using it.

\section{Materials and methods}

This study examined the role of personal time perspective and personal temporal experience in the occurrence of depressive, anxiogenic and somatization symptoms. A sample of 230 subjects of both sexes, aged between 25 and 60 years, was used. The following instruments were used: Temporal Experience Questionnaire, Time Perspective Inventory and the Symptom Checklist 90.

\section{Results}

Results show that subjects who are most likely to display frequent symptoms of depression, anxiety and/or somatization are those who are oriented towards negative aspects of their life in the past and/or view their present in a fatalistic or hedonistic way.

\section{Conclusions}

Furthermore, the occurrence of the mentioned symptoms is less probable for subjects who are oriented towards their future, organize their activities into time slots and perceive a high degree of control over their time. 